\title{
Pictorial Checklist of Agrobiont Spiders of Navsari Agricultural University, Navsari, Gujarat, India
}

\author{
J.N. Prajapati*, S.R. Patel and P.M. Surani \\ ${ }^{1}$ Department of Agricultural Entomology, N.M.C.A, NAU, Navsari, India \\ *Corresponding author
}

A B S T R A C T

\begin{abstract}
Keywords
Pictorial checklist, Agrobiont spiders,

Navsari,

biodiversity

Article Info

Accepted:

04 June 2018

Available Online:

A study on biodiversity of agrobiont spiders was carried out at N. M. College of Agriculture, Navsari Agricultural University (NAU) campus Navsari, Gujarat, India. A total 48 species of agrobiont spiders were recorded belonging to 34 genera and 12 families from different ecosystems i.e., paddy, sugarcane, maize, mango and banana. Among them 33.33 per cent species belongs to family Araneidae, 29.17 per cent from Salticidae, 8.33 per cent species belongs to family Oxyopidae, 6.25 per cent species belongs to family Clubionidae, 4.17 per cent species belongs to Tetragnathidae, Sparassidae as well as Theridiidae of each, whereas remaining 2.08 per cent species from Thomisidae, Uloboridae, Lycosidae, Hersiliidae, and Scytodidae of each and prepared the pictorial checklist of 48 species of agrobiont spiders.
\end{abstract}

10 July 2018

\section{Introduction}

Spiders are one of the most fascinating and diverse group of invertebrate animals on the earth. The population densities and species abundance of spider communities in agricultural fields can be as high as in natural ecosystems (Turnbull, 1973; Riechert, 1981 and Tanaka, 1989). Worldwide 45,557 spider species described (Uniyal et al., 2011) and are estimated to number $60,000-170,000$ species (Coddington and Levi, 1991). In numbers, 1686 species of 438 genera belongs to 60 families are recorded from India (Keswani et al., 2012). Total 415 species belong to 169 genera of 40 families are recorded from Gujarat state (Yadav et al., 2017). Spiders are considered to be of economic value to farmers as they play valuable role in pest management by consuming large number of prey in the agriculture fields without any damage to crops. To fill up the gaps in knowledge about different species of agrobiont spiders with their photographic catalogue in NAU campus, the present study was done.

\section{Materials and Methods}

The study was carried out at N. M. College of Agriculture, Navsari Agricultural University (NAU) campus Navsari, Gujarat, India from January 2017 to December 2017 under different agroecosystems. The ecosystems that are likely to support the spiders in the study 
area such as paddy, sugarcane, maize, mango and banana ecosystems were searched for agrobiont spiders. When a spider was located, it was photographed and collected by the hand picking method suggested by Tikader (1987). The identification of spiders was done with the help of Tikader (1977, 1980, 1982, 1987), Tikader and Biswas (1981) and Sebastian and Peter (2009). The taxonomy and nomenclature followed is as per the world spider catalogue by Platnick (2014). The pictorial checklist is a useful tool for the identification of agrobiont spiders in the state. Therefore, close up photographs of species and their behavioural patterns were captured with the help of digital camera. Live specimens from the field conditions were photographed, so that natural colouration and specific behavioural postures can be documented. When we have no camera, the spiders were collected and preserved and photographs of such preserved spiders were captured for documentation purpose in form of photographic catalogue. The picture of small spiders was taken by dissecting stereo-trinocular microscope having SCAPE software.

\section{Results and Discussion}

During the present study a total of 48 species of agrobiont spiders were recorded from different locations, belonging to 12 families. Among them 33.33 per cent species belonged to family Araneidae, 29.17 per cent from Salticidae, 8.33 per cent species belonged to family Oxyopidae, 6.25 per cent species belonged to family Clubionidae, 4.17 per cent species belonged to Tetragnathidae, Sparassidae as well as Theridiidae of each, whereas remaining 2.08 per cent species from Thomisidae, Uloboridae, Lycosidae, Hersiliidae and Scytodidae of each (Table 1 and Fig. 1). Ambily and Antony (2016) reported total 40 species of spiders belonging to 14 families from Kerala. Among all families, Araneidae was most dominant family followed by Salticidae.

Table.1 Species distribution of agrobiont spiders of different families in NAU campus

\begin{tabular}{|l|l|c|c|c|}
\hline Sr. No. & Family & Genera & $\begin{array}{c}\text { No. of } \\
\text { Species }\end{array}$ & \% Species \\
\hline 1. & Araneidae & 7 & 16 & 33.33 \\
\hline 2. & Salticidae & 14 & 14 & 29.17 \\
\hline 3. & Oxyopidae & 1 & 4 & 8.33 \\
\hline $\mathbf{4 .}$ & Clubionidae & 2 & 3 & 6.25 \\
\hline $\mathbf{5 .}$ & Tetragnathidae & 1 & 2 & 4.17 \\
\hline 6. & Sparassidae & 2 & 2 & 4.17 \\
\hline 7. & Theridiidae & 2 & 2 & 4.17 \\
\hline 8. & Thomisidae & 1 & 1 & 2.08 \\
\hline 9. & Uloboridae & 1 & 1 & 2.08 \\
\hline $\mathbf{1 0 .}$ & Lycosidae & 1 & 1 & 2.08 \\
\hline 11. & Hersiliidae & 1 & 1 & 2.08 \\
\hline 12. & Scytodidae & 1 & 1 & 2.08 \\
\hline Total & & 34 & 48 & 100 \\
\hline
\end{tabular}


Table.2 Pictorial checklist of agrobiont spiders of NAU campus

\begin{tabular}{|c|c|c|}
\hline $\begin{array}{l}\text { Sr. } \\
\text { No. }\end{array}$ & Spider species & Habitat \\
\hline \multicolumn{3}{|c|}{ I. Family: Araneidae } \\
\hline 1. & $\begin{array}{l}\text { Argiope anasuja (Thorell, 1887) } \\
\text { (Image 1) }\end{array}$ & $\begin{array}{l}\text { Paddy ecosystem, Sugarcane ecosystem, } \\
\text { Maize ecosystem, Banana ecosystem, } \\
\text { Garden, Wall of buildings }\end{array}$ \\
\hline 2. & $\begin{array}{l}\text { Argiope pulchella (Thorell, 1881) } \\
\text { (Image 2) }\end{array}$ & $\begin{array}{l}\text { Paddy ecosystem, Maize ecosystem, } \\
\text { Banana ecosystem, Garden }\end{array}$ \\
\hline 3. & $\begin{array}{l}\text { Argiope sp. } \\
\text { (Image 3) }\end{array}$ & $\begin{array}{l}\text { Paddy ecosystem, Banana ecosystem, } \\
\text { Garden }\end{array}$ \\
\hline 4. & $\begin{array}{l}\text { Argiope aemula (Walckenaer, 1841) } \\
\text { (Image 4) }\end{array}$ & Paddy ecosystem, Garden \\
\hline 5. & $\begin{array}{l}\text { Neoscona mukerjei (Tikader, 1980) } \\
\text { (Image 5) }\end{array}$ & $\begin{array}{l}\text { Paddy ecosystem, Sugarcane ecosystem, } \\
\text { Maize ecosystem, Mango ecosystem, } \\
\text { Banana ecosystem, Dense area, } \\
\text { Vegetable crops }\end{array}$ \\
\hline 6. & $\begin{array}{l}\text { Neoscona theisi (Walckenaer, 1842) } \\
\text { (Image 6) }\end{array}$ & $\begin{array}{l}\text { Paddy ecosystem, Sugarcane ecosystem, } \\
\text { Maize ecosystem, Mango ecosystem, } \\
\text { Banana ecosystem, Vegetable crops }\end{array}$ \\
\hline 7. & $\begin{array}{l}\text { Neoscona bengalensis (Tikader and Bal, } \\
\text { 1981) (Image 7) }\end{array}$ & $\begin{array}{l}\text { Paddy ecosystem, Mango ecosystem, } \\
\text { Banana ecosystem, Arjun, Garden }\end{array}$ \\
\hline 8. & $\begin{array}{l}\text { Neoscona vigilans (Blackwall, 1865) } \\
\text { (Image } 8 \text { ) }\end{array}$ & $\begin{array}{l}\text { Paddy ecosystem, Sugarcane ecosystem, } \\
\text { Garden }\end{array}$ \\
\hline 9. & $\begin{array}{l}\text { Neoscona sp. } 1 \\
\text { (Image 9) }\end{array}$ & $\begin{array}{l}\text { Paddy ecosystem, Sugarcane ecosystem, } \\
\text { Maize ecosystem, Garden, Vegetable } \\
\text { crops }\end{array}$ \\
\hline 10. & $\begin{array}{l}\text { Neoscona sp. } 2 \\
\text { (Image 10) }\end{array}$ & $\begin{array}{l}\text { Paddy ecosystem, Maize ecosystem, } \\
\text { Mango ecosystem, Cruciferous crops, } \\
\text { Garden }\end{array}$ \\
\hline 11. & $\begin{array}{l}\text { Pasilobus sp. } \\
\text { (Image 11) }\end{array}$ & $\begin{array}{l}\text { Paddy ecosystem, Sugarcane ecosystem, } \\
\text { Garden }\end{array}$ \\
\hline 12. & $\begin{array}{l}\text { Cyclosa confraga (Thorell, 1892) } \\
\text { (Image 12) }\end{array}$ & $\begin{array}{l}\text { Paddy ecosystem, Maize ecosystem, } \\
\text { Mango ecosystem, Banana ecosystem, } \\
\text { Vegetable crops }\end{array}$ \\
\hline 13. & $\begin{array}{l}\text { Cyclosa sp. } \\
\text { (Image 13) }\end{array}$ & $\begin{array}{l}\text { Paddy ecosystem, Maize ecosystem, } \\
\text { Garden }\end{array}$ \\
\hline 14. & $\begin{array}{l}\text { Cyrtophora cicatrosa (Stoliczka, 1869) } \\
\text { (Image 14) }\end{array}$ & $\begin{array}{l}\text { Paddy ecosystem, Sugarcane ecosystem, } \\
\text { Banana ecosystem, Garden }\end{array}$ \\
\hline 15. & $\begin{array}{l}\text { Eriovixia sp. } \\
\text { (Image 15) }\end{array}$ & $\begin{array}{l}\text { Mango ecosystem, Banana ecosystem, } \\
\text { Garden, Fruit trees }\end{array}$ \\
\hline 16. & $\begin{array}{l}\text { Larinia sp. } \\
\text { (Image 16) }\end{array}$ & $\begin{array}{l}\text { Paddy ecosystem, Sugarcane ecosystem, } \\
\text { Maize ecosystem, Wall of buildings, }\end{array}$ \\
\hline
\end{tabular}




\begin{tabular}{|c|c|c|}
\hline \multirow{2}{*}{\multicolumn{3}{|c|}{ II. Family: Salticidae }} \\
\hline & & \\
\hline 17. & $\begin{array}{l}\text { Carrhotus viduus (Koch, C. L., 1846) } \\
\text { (Image 17) }\end{array}$ & $\begin{array}{l}\text { Paddy ecosystem, Sugarcane ecosystem, } \\
\text { Maize ecosystem, Mango ecosystem, } \\
\text { Banana ecosystem, Wall of buildings }\end{array}$ \\
\hline 18. & $\begin{array}{l}\text { Epeus indicus (Proszynski, 1992) } \\
\text { (Image 18) }\end{array}$ & Maize ecosystem, Broad leaves crops \\
\hline 19. & $\begin{array}{l}\text { Plexippus paykulli (Audouin, 1826) } \\
\text { (Image 19) }\end{array}$ & $\begin{array}{l}\text { Mango ecosystem, Banana ecosystem, } \\
\text { Walls of buildings, Bark of trees }\end{array}$ \\
\hline 20. & $\begin{array}{l}\text { Phintella vittata (Koch, C. L., 1846) } \\
\text { (Image 20) }\end{array}$ & $\begin{array}{l}\text { Sugarcane ecosystem, Maize ecosystem, } \\
\text { Banana ecosystem, Papaya, Marigold }\end{array}$ \\
\hline 21. & $\begin{array}{l}\text { Chrysilla volupe (Karsch, 1879) } \\
\text { (Image 21) }\end{array}$ & $\begin{array}{l}\text { Paddy ecosystem, Sugarcane ecosystem, } \\
\text { Maize ecosystem, Banana ecosystem, } \\
\text { Grasses, Marigold }\end{array}$ \\
\hline 22. & $\begin{array}{l}\text { Unknown sp.1 (Salticidae) } \\
\text { (Image 22) }\end{array}$ & $\begin{array}{l}\text { Banana ecosystem, Walls of buildings, } \\
\text { Dry grasses }\end{array}$ \\
\hline 23. & $\begin{array}{l}\text { Pristobaeus sp. } \\
\text { (Image 23) }\end{array}$ & $\begin{array}{l}\text { Sugarcane ecosystem, Banana } \\
\text { ecosystem, Dry grasses }\end{array}$ \\
\hline 24. & $\begin{array}{l}\text { Evarcha falcate (Clerck, 1757) } \\
\text { (Image 24) }\end{array}$ & Sugarcane ecosystem, Walls of buildings \\
\hline 25. & $\begin{array}{l}\text { Unknown sp.2 (Salticidae) } \\
\text { (Image 25) }\end{array}$ & $\begin{array}{l}\text { Paddy ecosystem, Sugarcane ecosystem, } \\
\text { Garden }\end{array}$ \\
\hline 26. & $\begin{array}{l}\text { Cosmophasis sp. } \\
\text { (Image 26) }\end{array}$ & Banana ecosystem, Walls of buildings \\
\hline 27. & $\begin{array}{l}\text { Telamonia dimidiata (Simon, 1899) } \\
\text { (Image 27) }\end{array}$ & $\begin{array}{l}\text { Sugarcane ecosystem, Maize ecosystem, } \\
\text { Cotton, Mulberry }\end{array}$ \\
\hline 28. & $\begin{array}{l}\text { Menemerus bivittatus (Dufour, 1831) } \\
\text { (Image 28) }\end{array}$ & $\begin{array}{l}\text { Mango ecosystem, Banana ecosystem, } \\
\text { Walls of buildings }\end{array}$ \\
\hline 29. & $\begin{array}{l}\text { Siler sp. } \\
\text { (Image 29) }\end{array}$ & Maize ecosystem, Garden \\
\hline 30. & $\begin{array}{l}\text { Rhene sp. } \\
\text { (Image 30) }\end{array}$ & Paddy ecosystem, Jasmine \\
\hline \multicolumn{3}{|c|}{ III. Family: Oxyopidae } \\
\hline 31. & $\begin{array}{l}\text { Oxyopes javanus (Thorell, 1887) } \\
\text { (Image } 31 \text { ) }\end{array}$ & $\begin{array}{l}\text { Paddy ecosystem, Sugarcane ecosystem, } \\
\text { Maize ecosystem, Mango ecosystem, } \\
\text { Banana ecosystem, Marigold, Garden }\end{array}$ \\
\hline 32. & $\begin{array}{l}\text { Oxyopes sunandae (Tikader, 1970) } \\
\text { (Image 32) }\end{array}$ & $\begin{array}{l}\text { Paddy ecosystem, Sugarcane ecosystem, } \\
\text { Maize ecosystem, Marigold }\end{array}$ \\
\hline 33. & $\begin{array}{l}\text { Oxyopes sp. } \\
\text { (Image 33) }\end{array}$ & $\begin{array}{l}\text { Paddy ecosystem, Sugarcane ecosystem, } \\
\text { Maize ecosystem, Banana ecosystem, } \\
\text { Cotton, Garden }\end{array}$ \\
\hline 34. & $\begin{array}{l}\text { Oxyopes birmanicus (Thorell, 1887) } \\
\text { (Image 34) }\end{array}$ & $\begin{array}{l}\text { Paddy ecosystem, Sugarcane ecosystem, } \\
\text { Maize ecosystem, Marigold }\end{array}$ \\
\hline
\end{tabular}




\begin{tabular}{|c|c|c|}
\hline 35. & $\begin{array}{l}\text { Clubiona sp. } \\
\text { (Image 35) }\end{array}$ & $\begin{array}{l}\text { Paddy ecosystem, Sugarcane ecosystem, } \\
\text { Maize ecosystem, Banana ecosystem, } \\
\text { Rolled leaves }\end{array}$ \\
\hline 36. & $\begin{array}{l}\text { Clubiona drassodes (Cambridge, O. P., 1874) } \\
\text { (Image 36) }\end{array}$ & $\begin{array}{l}\text { Paddy ecosystem, Sugarcane ecosystem, } \\
\text { Maize ecosystem, Banana ecosystem, } \\
\text { Rolled leaves }\end{array}$ \\
\hline 37. & $\begin{array}{l}\text { Cheiracanthium punctorium } \\
\text { (Image } 37 \text { ) }\end{array}$ & $\begin{array}{l}\text { Paddy ecosystem, Banana ecosystem, } \\
\text { Rolled leaves, Garden }\end{array}$ \\
\hline \multicolumn{3}{|c|}{ V. Family: Tetragnathidae } \\
\hline 38. & $\begin{array}{l}\text { Tetragnatha mandibulata (Walckenaer, 1842) } \\
\text { (Image 38) }\end{array}$ & Paddy ecosystem, Near vicinity of water \\
\hline 39. & $\begin{array}{l}\text { Tetragnatha sp. } \\
\text { (Image 39) }\end{array}$ & Paddy ecosystem, Near vicinity of water \\
\hline \multicolumn{3}{|c|}{ VI. Family: Sparassidae } \\
\hline 40. & $\begin{array}{l}\text { Heteropoda venatoria (Linnaeus, 1767) } \\
\text { (Image 40) }\end{array}$ & $\begin{array}{l}\text { Paddy ecosystem, Sugarcane ecosystem, } \\
\text { Maize ecosystem, Anthurium, Fruit } \\
\text { boxes, Houses, Warm places }\end{array}$ \\
\hline 41. & $\begin{array}{l}\text { Olios } s p . \\
\text { (Image } 41 \text { ) }\end{array}$ & $\begin{array}{l}\text { Paddy ecosystem, Maize ecosystem, } \\
\text { Warm places, Houses }\end{array}$ \\
\hline \multicolumn{3}{|c|}{ VII. Family: Theridiidae } \\
\hline 42. & $\begin{array}{l}\text { Theridion } \mathrm{sp} . \\
\text { (Image } 42 \text { ) }\end{array}$ & $\begin{array}{l}\text { Sugarcane ecosystem, Maize ecosystem, } \\
\text { Garden }\end{array}$ \\
\hline 43. & $\begin{array}{l}\text { Steatoda } s p \text {. } \\
\text { (Image } 43 \text { ) }\end{array}$ & $\begin{array}{l}\text { Mango ecosystem, Banana ecosystem, } \\
\text { Gerbera, Marigold }\end{array}$ \\
\hline \multicolumn{3}{|c|}{ VIII. Family: Thomisidae } \\
\hline 44. & $\begin{array}{l}\text { Thomisus sp. } \\
\text { (Image 44) }\end{array}$ & $\begin{array}{l}\text { Paddy ecosystem, Sugarcane ecosystem, } \\
\text { Maize ecosystem, Marigold, Gerbera }\end{array}$ \\
\hline \multicolumn{3}{|c|}{ IX. Family: Uloboridae } \\
\hline 45. & $\begin{array}{l}\text { Uloborus plumipes (Lucas, 1846) } \\
\text { (Image 45) }\end{array}$ & $\begin{array}{l}\text { Mango ecosystem, Banana ecosystem, } \\
\text { Gerbera }\end{array}$ \\
\hline \multicolumn{3}{|c|}{ X. Family: Lycosidae } \\
\hline 46. & $\begin{array}{l}\text { Lycosa sp. } \\
\text { (Image 46) }\end{array}$ & Paddy ecosystem, Wet land \\
\hline \multicolumn{3}{|c|}{ XI. Family: Hersiliidae } \\
\hline 47. & $\begin{array}{l}\text { Hersilia savignyi (Lucas, 1836) } \\
\text { (Image 47) }\end{array}$ & Mango ecosystem, Tree-trunks \\
\hline \multicolumn{3}{|c|}{ XII. Family: Scytodidae } \\
\hline 48. & $\begin{array}{l}\text { Scytodes thoricica (Latreille, 1802) } \\
\text { (Image 48) }\end{array}$ & $\begin{array}{l}\text { Paddy ecosystem, Sugarcane ecosystem, } \\
\text { Maize ecosystem, Marigold, Jasmine, } \\
\text { Stones on ground }\end{array}$ \\
\hline
\end{tabular}


Fig.1 Distribution of different families in Araneae Order

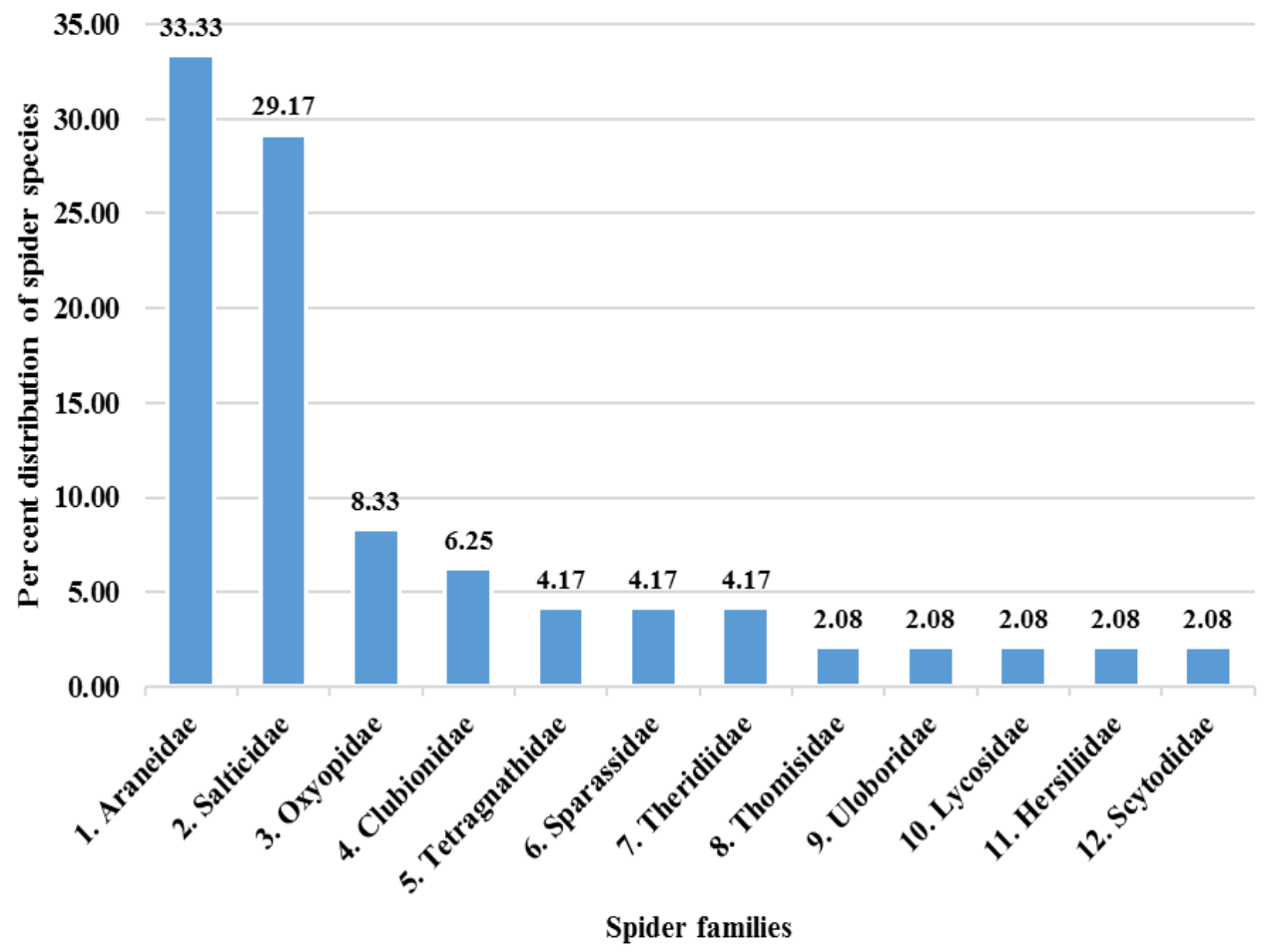

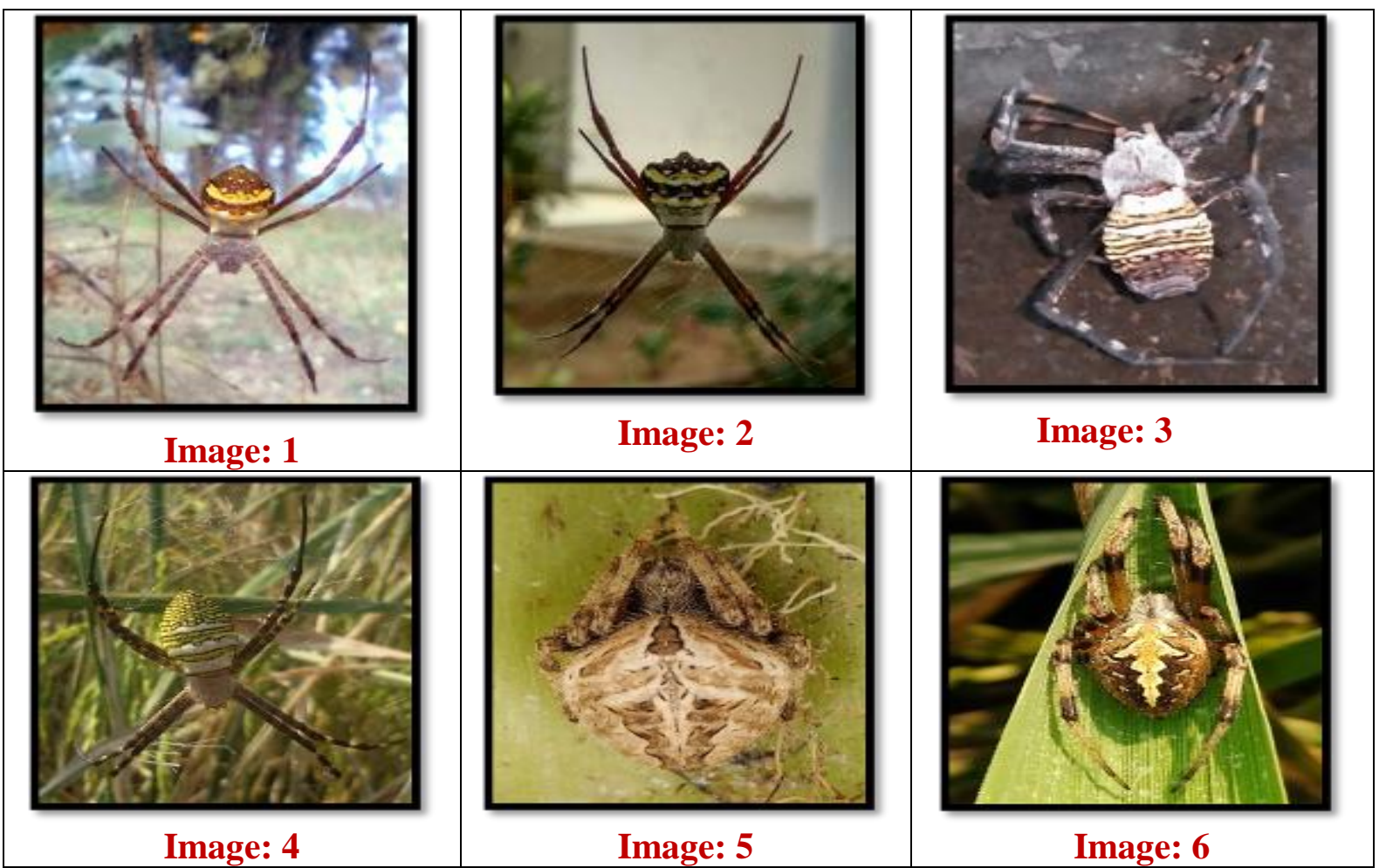




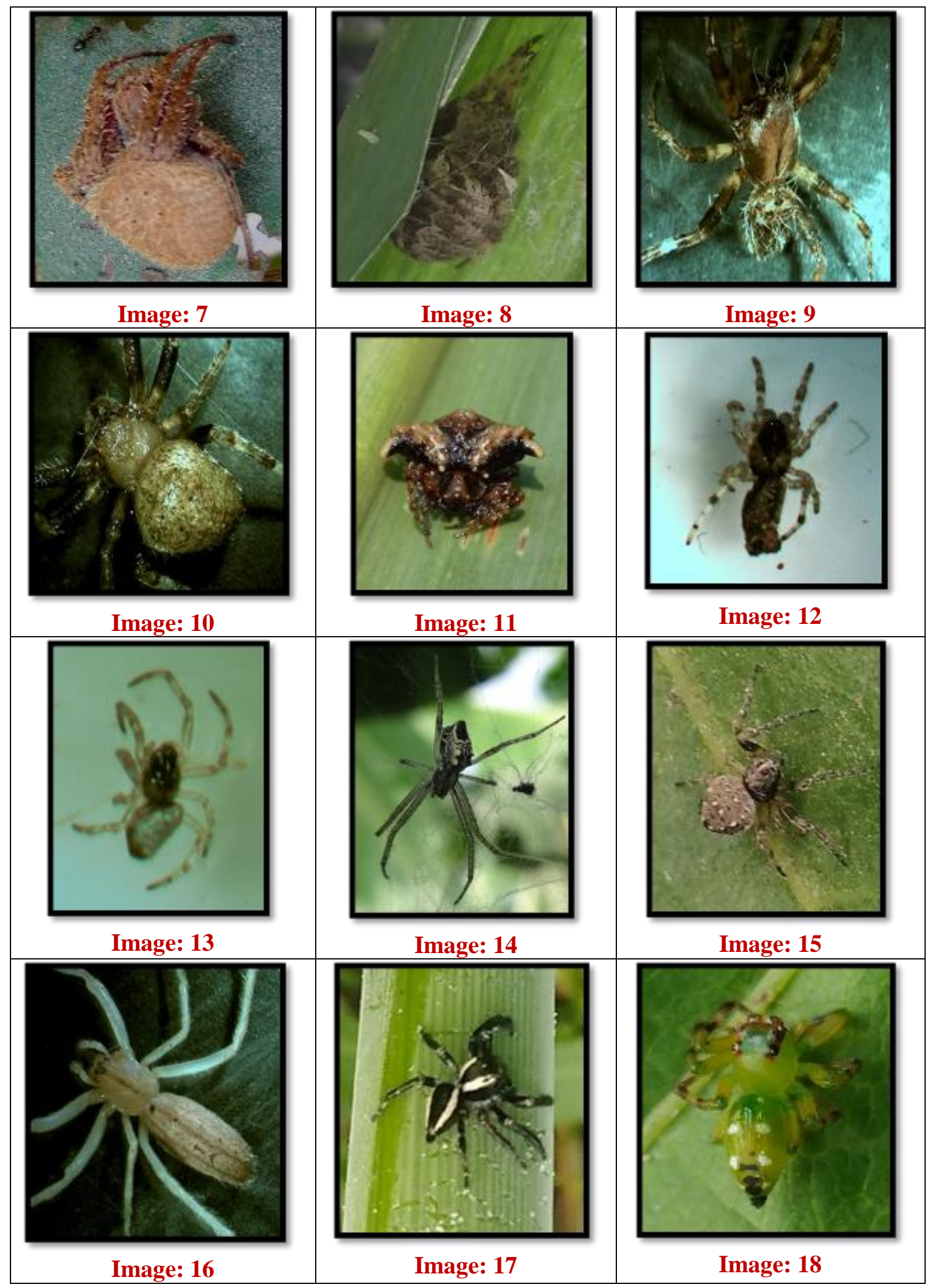




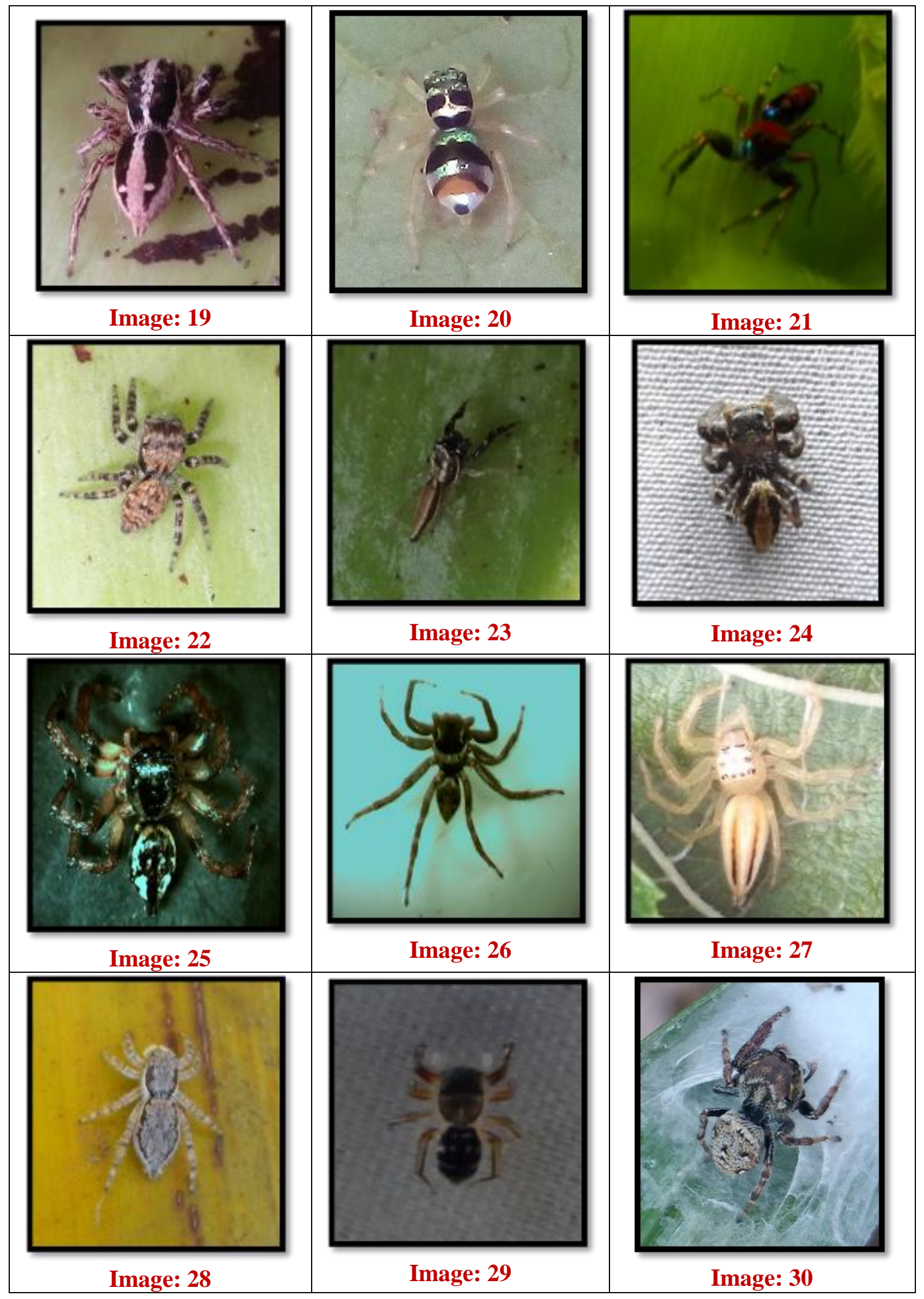




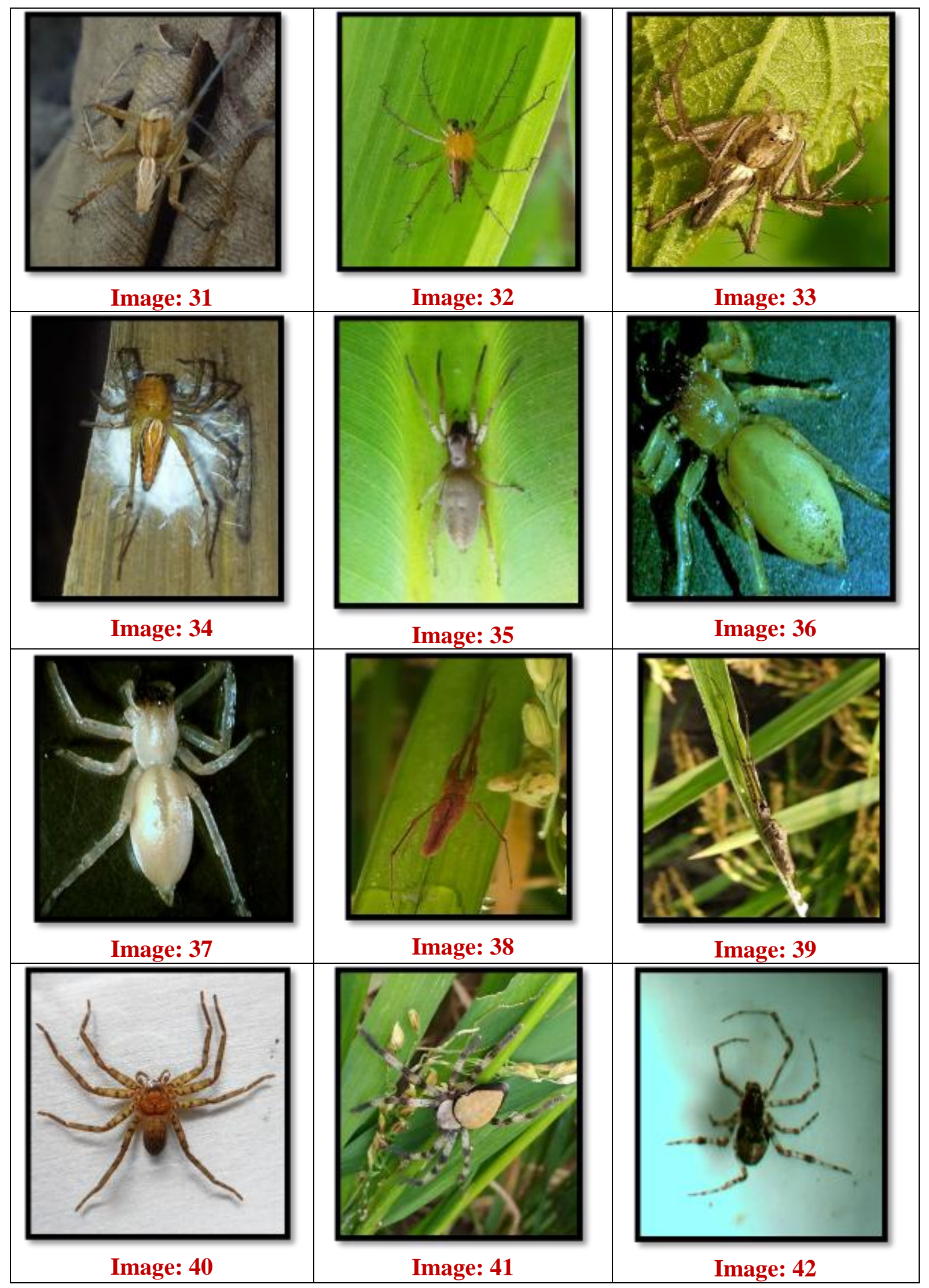




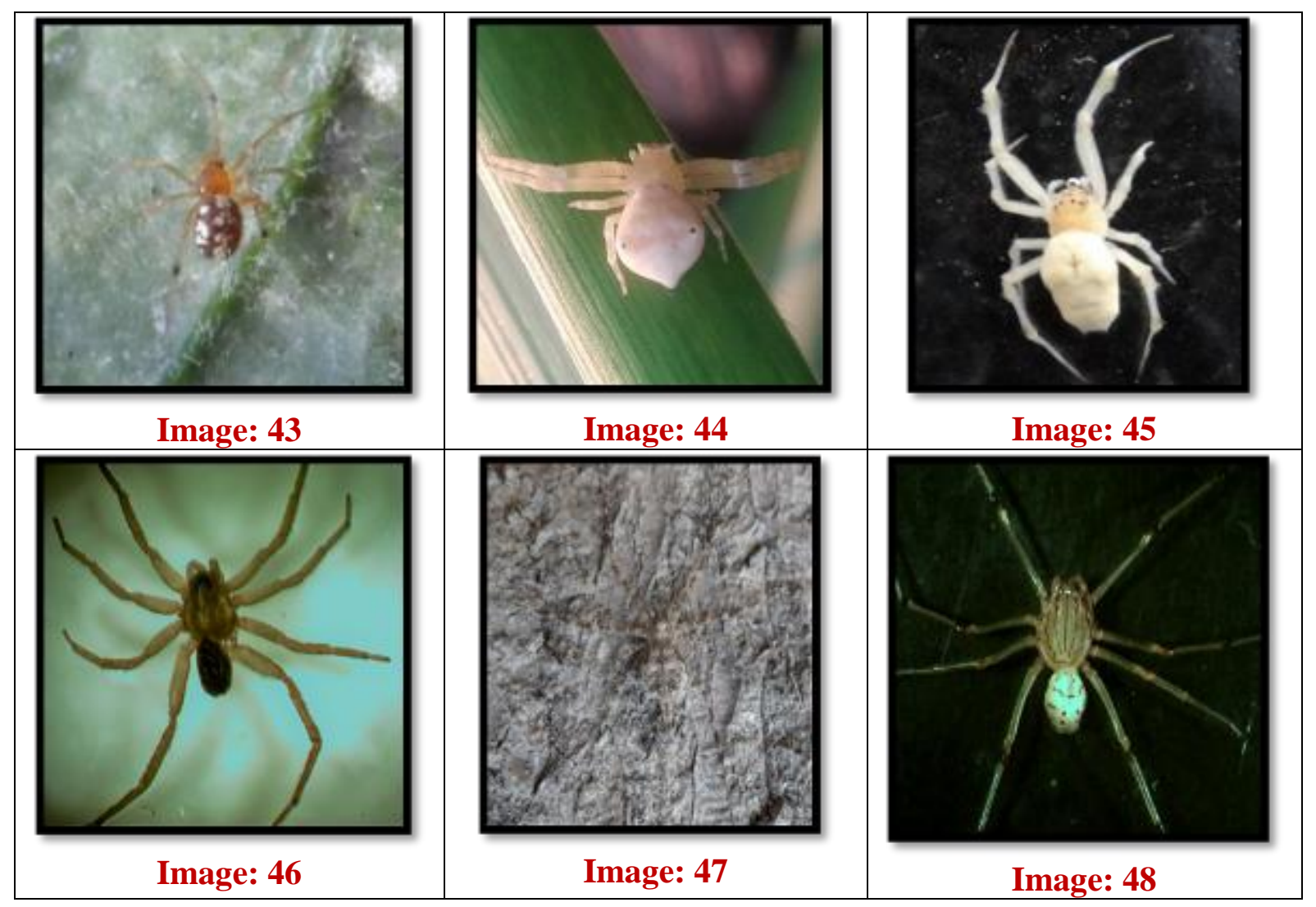

Under the present study Araneidae was the most dominant family comprising of seven genera and 16 species with 33.33 per cent species distribution. This may be more or less in accordance with the earlier work. Further, More (2015) from Maharashtra also recorded Araneidae as one of the most dominant family, thus closely support the present findings. In the present investigation, a total of 48 species of agrobiont spiders in 34 genera belonging to 12 families were recorded and the pictorial checklist of different agrobiont spiders from the study area was prepared (Table 2).

The checklist of the Araneae of different countries/continents/ecozones were published in recent past by several authors, like Gajbe (2003), Dandria et al., (2005), Siliwal et al., (2005), Namkung et al., (2009), Ursani and Soomro (2010), Khan (2011), Perveen and Jamal (2012), Sial et al., (2012), Adarsh and Nameer (2015), Lawania and Trigunayat
(2015), Perveen and Khan (2015), Adarsh and Nameer (2016), Ghazanfar et al., (2016) and Prajapati et al., (2016). The present compilation was also more or less similar in accordance with the earlier checklist, as most of the families and species were found in these checklist. In the present study the spider families Araneidae, Salticidae, Oxyopidae, Clubionidae, Tetragnathidae, Sparassidae and Theridiidae were commonly found in Navsari Agricultural University.

In conclusion, the pictorial checklist of total 48 species of agrobiont spiders were recorded which belongs to 34 genera and 12 families from different habitats with their taxonomic position.

\section{Acknowledgement}

The authors are thankful to Prof. Ramesh Thumar, Assistant Professor, Department of Zoology, B. P. Baria Science College, 
Navsari, Gujarat for identifying spider species and valuable suggestions.

\section{References}

Adarsh, C. K. and Nameer, P. O. (2015). Spiders of Kerala Agricultural University Campus, Thrissur, Kerala, India. Journal of Threatened Taxa, 7(15): 8288-8295.

Adarsh, C. K. and Nameer, P. O. (2016). A preliminary checklist of spiders (Araneae: Arachnida) in Chinnar Wildlife Sanctuary, Western Ghats, India. Journal of Threatened Taxa, 8(4): 8703-8713.

Ambily, C. B. and Antony, A. (2016). Diversity and distribution of spiders in agro ecosystem of Ernakulum District, Kerala. The Journal of Zoology Studies, 3(5): 73-77.

Coddington, J. A. and Levi, H. W. (1991). Systematics and evolution of spiders (Araneae). Annual Review of Ecology and Systematics, 22: 565-592.

Dandria, D., Falzon, V. and Henwood, J. (2005). The current knowledge of the spider fauna of the Maltese Islands with the addition of some new records (Arachnida: Araneae). The Central Mediterranean Naturalist, 4(2): 121129.

Gabje, P. (2003). Checklist of spiders (Arachnida: Araneae) of Madhya Pradesh and Chhattisgarh. Zoos' Print Journal, 18(10): 1223-1226.

Ghazanfar, M., Hussain, M., Hashim, M. and Mustafa fahid, A. U. (2016). Check list of spider (Araneae) fauna of Pakistan: A review. Journal of Entomology and Zoology Studies, 4(1): 245-256.

Keswani, S., Hadole, P. and Rajoria, A. (2012). Check list of Spiders (Arachnida: Araneae) from India, Indian Journal of Arachnology, 1(1):
1-129.

Khan, A. A. (2011). Spider fauna (Arachnida: Araneae) in Horticultural ecosystem of Kashmir. Animal Diversity, Natural History and Conservation, 1: 313-338.

Lawania, K. K. and Trigunayat, M. M. (2015). A comparative study of the spider (Araneae) fauna in Keoladeo National Park (KNP), Nahargarh Wildlife Sanctuary (NWS) and SurSarovar Bird Sanctuary (SBS), India. Mun. Ent. Zool., 10(2): 435-440.

More S. B. (2015). Inventorization of spider diversity from Vakoba, Devrai region of Radhanagari Wildlife Sanctuary. International Journal of Science and Research, 4(6): 179-181.

Namkung, J., Yoo, J. S., Lee, S. Y., Lee, J. H., Paek, W. K. and Kim, S. T. (2009). Bibliographic check list of Korean spiders (Arachnida: Araneae) ver. 2010. Journal of Korean Nature, 2(3): 191-285.

Perveen, F. and Jamal, A. (2012). Checklist of spider fauna of FR Peshawar, FATA, Pakistan. Arthropods, 1(1): 35-39.

Perveen, F. and Khan, N. (2015). Checklist of the first recorded spider fauna in Sheringal, Khyber Pakhtunkhwa, Pakistan. Advances in Entomology, 3: 94-100.

Platnick, N. I. (2014). The world spider catalog, Version 15.0. American Museum of Natural History http://research.amnh.org/entomology/s piders/catalog/index. Accessed on 25 January 2017.

Prajapati, D. A., Patel, K. R., Munjpara, S. B., Chettiar, S. S. and Jhala, D. D. (2016). Spiders (Arachnida: Araneae) of Gujarat University Campus, Ahmedabad, India with additional description of Eilica tikaderi (Platnick, 1976). Journal of Threatened Taxa, 8(11): 9327-9333.

Riechert, S. E. (1981). The consequences of 
being territorial: Spiders, a case study. American Naturalist, 117: 871-892.

Sebastian, P. A. and Peter, K. V. (2009). Spiders of India. Universities Press (India) Private Limited, Hyderabad, $614 \mathrm{pp}$.

Sial, N., Ruby, T., Malik, S. and Mushtaq, S. (2012). A checklist of the spiders of Cholistan and neighbouring areas. Pak. J. Agri. Sci., 49(3): 335-338.

Siliwal, S., Molur, S. and Biswas, B. K. (2005). Indian spiders (Arachnida: Araneae): Updated Checklist 2005. Zoos' Print Journal, 20(10): 19992049.

Tanaka, K. (1989). Movement of the spiders in arable land. Plant Protection, 43(1): 34-39.

Tikader, B. K. (1977). Studies on spider fauna of Andaman and Nicobar Islands India Ocean. Records of the Zoological Survey of India, 72: 153-212.

Tikader, B. K. (1980). Fauna of India Araneae: Spiders, Vol. I (Araneidae and Gnaphosidae). Zoological Survey of India, $448 \mathrm{pp}$.

Tikader, B. K. (1982). Fauna of India -
Araneae: Spiders, Vol. II (Thomisidae and Lycosidae). Zoological Survey of India, $533 \mathrm{pp}$.

Tikader, B. K. (1987). Hand book of Indian Spiders. Zoological Survey of India, $251 \mathrm{pp}$.

Tikader, B. K. and Biswas, B. (1981). Spider Fauna of Calcutta and Vicinity. Zoological survey of India, Kolkata, $149 \mathrm{pp}$.

Turnbull, A. L. (1973). Ecology of the true spiders (Araneomorphae). Annual Review of Entomology, 18: 305-348.

Uniyal, V. P., Sivakumar, K. and Quasin, S., (2011). Diversity of Spiders in Nanda Devi Biosphere Reserve. Wildlife Institute of India, Dehradun. (DST Project Completion Report).

Ursani, T. J. and Soomro, N. M. (2010). Check-list of spider fauna of Sindh Province, Pakistan. Pakistan Entomologist, 32(1): 18-23.

Yadav, A., Solanki, R., Siliwal, M. and Kumar, D. (2017). Spiders of Gujarat: a preliminary checklist. Journal of Threatened Taxa, 9(9): 10697-10716.

\section{How to cite this article:}

Prajapati, J.N., S.R. Patel and Surani, P.M. 2018. Pictorial Checklist of Agrobiont Spiders of Navsari Agricultural University, Navsari, Gujarat, India. Int.J.Curr.Microbiol.App.Sci. 7(07): 409-420. doi: https://doi.org/10.20546/ijcmas.2018.707.050 